\title{
A rare functional variant of SHARPIN attenuates the inflammatory response and associates with increased risk of late-onset Alzheimer's disease
}

\author{
Yuya Asanomi ${ }^{1}$, Daichi Shigemizu ${ }^{1,2,3}$, Akinori Miyashita ${ }^{4}$, Risa Mitsumori ${ }^{1}$, Taiki Mori ${ }^{1}$, Norikazu Hara ${ }^{4}$, Kaoru Ito ${ }^{5}$, \\ Shumpei Niida ${ }^{6}$, Takeshi Ikeuchi ${ }^{4}$ and Kouichi Ozaki ${ }^{1,5^{*}}$
}

\begin{abstract}
Background: Late-onset Alzheimer's disease (LOAD), the most common form of dementia, results from complicated interactions among multiple environmental and genetic factors. Despite recent advances in genetic analysis of LOAD, more than half of the heritability for the disease remains unclear. Although genetic studies in Caucasians found rare risk variants for LOAD with large effect sizes, these variants are hardly detectable in the Japanese population.

Methods: To identify rare variants possibly explaining part of the genetic architecture for LOAD in Japanese people, we performed whole-exome sequencing analyses of 202 LOAD individuals without the APOE $\varepsilon 4$ risk allele, a major genetic factor for LOAD susceptibility. We also implemented in vitro functional analyses of the variant(s) to reveal possible functions associated with LOAD risk.

Results: Via step-by-step selection of whole-exome variants, we found seven candidate risk variants. We then conducted a case-control association study in a large Japanese cohort consisting of 4563 cases and 16,459 controls. We finally identified a rare nonsynonymous variant, rs572750141 (NM_030974.3:p.Gly186Arg), in SHARPIN that was potentially associated with increased risk of $\operatorname{LOAD}$ (corrected $P=8.05 \times 10^{-5}$, odds ratio $=6.1$ ). The amino acid change in SHARPIN resulted in aberrant cellular localization of the variant protein and attenuated the activation of NF-KB, a central mediator of inflammatory and immune responses.

Conclusions: Our work identified a rare functional SHARPIN variant as a previously unknown genetic risk factor for LOAD. The functional alteration in SHARPIN induced by the rare coding variant is associated with an attenuated inflammatory/immune response that may promote LOAD development.
\end{abstract}

Keywords: Alzheimer's disease, Inflammation, Rare functional variant, Genetic risk factor, SHARPIN

\section{Background}

Dementia is one of the leading causes of death in many countries. According to a nationwide survey in 2012, more than $15 \%$ of people aged 65 and older in Japan have dementia. (Saji et al., 2019) Currently, there is no effective therapy for LOAD, despite numerous clinical

\footnotetext{
*Correspondence: ozakikk@ncgg.go.jp; ozakikk@riken.jp

'Division for Genomic Medicine, Medical Genome Center, National Center for Geriatrics and Gerontology, Obu, Aichi, Japan

${ }^{5}$ Laboratory for Cardiovascular Diseases, RIKEN Center for Integrative Medical Sciences, Yokohama, Japan

Full list of author information is available at the end of the article
}

trials of pharmaceutical approaches. Immediate measures against dementia are therefore desired.

The most common form of dementia is Alzheimer's disease $(\mathrm{AD})$. $\mathrm{AD}$ includes the familial form (early-onset $\mathrm{AD}$ ) and a more common sporadic form (late-onset $\mathrm{AD}$ [LOAD]). (Van Cauwenberghe et al., 2016) LOAD results from complex interactions among multiple genetic and environmental factors. (Bagyinszky et al., 2014; Scheltens et al., 2016) A large twin study confirmed that the heritability for $\mathrm{AD}$ is high $\left(h^{2}=58-79 \%\right)$. (Gatz et al., 2006) A large-scale genome-wide association study (GWAS) meta-analysis found more than 30 genomic loci associated 
with the risk of LOAD. (Lambert et al., 2013) However, the $A P O E \& 4$ allele is the only genetic risk factor for LOAD consistently observed in all races, with the other loci showing smaller effects with modest odds ratios. Accordingly, much of the heritability of LOAD remains unclear.

Recently, two different Caucasian cohort studies identified a rare nonsynonymous variant (rs75932628: p.Arg47His) in TREM2 as a strong risk factor for LOAD with odds ratio of 2.92 (Jonsson et al., 2013) and 4.59. (Guerreiro et al., 2013) By focusing on this variant, the role of TREM2 in microglia and its association with LOAD were indicated by using a mouse model of AD. (Wang et al., 2015) However, the minor allele frequency (MAF) of rs75932628 in Japanese LOAD patients was estimated to be just 0.00023 (Miyashita et al., 2014), whereas the population frequency of this variant in Iceland was 0.0063. (Jonsson et al., 2013) Furthermore, in addition to TREM2, rare coding variants have been reported in PLCG2 and ABI3. (Sims et al., 2017) However, according to the Genome Aggregation Database (gnomAD, http://gnomad.broadinstitute.org/), none of these variants are found in East Asian individuals. Therefore, we performed a systematic search to identify rare variants that might explain some of the genetic architecture for LOAD in Japanese people.

In this study, we attempted to discover rare genetic variants associated with the risk of LOAD based on whole-exome sequencing analyses of 202 Japanese individuals with LOAD who do not have the APOE $\varepsilon 4$ risk allele. We identified a rare nonsynonymous variant with strong effect in SHARPIN. We also demonstrated that the amino acid substitution of the novel rare risk variant of SHAR$P I N$ affected its cellular localization and NF- $\mathrm{kB}$ activity. Our functional studies of the SHARPIN variant thus suggest that this variation promotes the pathogenesis of LOAD by affecting inflammatory/immune pathways.

\section{Methods}

\section{Study population}

Clinical information, which included age, sex, diagnosis, and $A P O E \& 4$ allele genotype, and the genomic DNA of LOAD patients and normal cognitive function (NC) control participants were obtained from the National Center for Geriatrics and Gerontology (NCGG) Biobank. The NCGG Biobank is one of the facilities belonging to the National Center Biobank Network (NCBN; https:// ncbiobank.org/en/home.php). It has been running since 2012. The participants were recruited from NCGG hospital, which is located in Obu city, and the other nearby medical institutes. LOAD was diagnosed at NCGG hospital according to the diagnostic criteria developed by the National Institute on Aging and the Alzheimer's Association (NIA-AA). (Albert et al., 2011; McKhann et al., 2011) We also applied regional cohort samples of elderly adults ( $\geq 65$ years) in Aichi prefecture, stored in the NCGG Biobank, as general Japanese population samples for the association study. The demographic features of the NCGG samples applied in the exome sequencing and genotyping are shown in Additional file 1: Tables S1-S3. In the first cohort of the association study of two variants, rs572750141 and rs531355933, we also included 7345 DNA samples recruited at RIKEN and public whole-genome sequence data of 3554 Japanese individuals (3.5KJPN, Integrative Japanese Genome Variation; https://ijgvd.megabank.tohoku.ac.jp/) from Tohoku Medical Megabank Organization (TMM).

For the second cohort of the association study of the two variants identified, we obtained 2180 LOAD cases and 2486 controls recruited from Niigata University (Additional file 1: Table S4); their demographics and the clinical criteria used for the diagnosis of $\mathrm{AD}$ are described in a previous report. (Miyashita et al., 2014) All individuals are Japanese and provided written informed consent, and the study was performed with the approval of the ethics review board at NCGG and of each institute.

\section{Exome sequencing and variant calling}

For whole-exome sequencing, we used two kinds of next-generation sequencers, a HiSeq 2500 (Illumina, San Diego, CA) and an IonProton (Thermo Fisher Scientific, Waltham, MA). Genomic DNA samples were quantified using the Quant-iT ${ }^{\mathrm{m}}$ PicoGreen $^{\circ}$ dsDNA Assay Kit or Qubit dsDNA HS Assay Kit (Thermo Fisher Scientific).

For HiSeq, exome libraries were prepared with the Nextera Rapid Capture Expanded Exome Kit (Illumina) or SureSelect Human All Exon V6 (Agilent Technologies, Santa Clara, CA) according to the manufacturers' protocols. Enriched exome libraries were analyzed by using an Agilent 4200 TapeStation (Agilent) or DNA Fragment Analyzer (Advanced Analytical, Ankeny, IA). The libraries were then sequenced on a HiSeq 2500 system. Paired-end reads of 125 nucleotides were sequenced by using the HiSeq PE Cluster Kit v4 cBot and HiSeq SBS Kit v4. Data processing was performed by using a Resequence/Exome analysis pipeline (Amelieff, Tokyo, Japan). All software in the pipeline was used with properly tuned default settings. Briefly, for use in this pipeline, raw data from the HiSeq system were converted to the FASTQ format with bcl2fastq (Illumina). At the beginning of the pipeline, the FASTQ-formatted files were cleaned up with QCleaner software (Amelieff). Then, sequence reads were mapped to the human genome (hg19) using a BWA algorithm (http://bio-bwa.sourceforge.net/). Duplicated reads were removed by applying Picard (http://broadinstitute.github. io/picard/). Variant calling for single-nucleotide variants (SNVs) and indels was performed with GATK (https:// www.broadinstitute.org/gatk/), and variants were outputted in VCF format. 
For the IonProton sequencer, exome libraries were prepared with the Ion TargetSeq Exome Enrichment Kit or Ion AmpliSeq Exome RDY Kit (Thermo Fisher Scientific) according to the manufacturer's protocol. The enriched exome libraries were analyzed by real-time PCR with the GeneRead Library Quant Kit (QIAGEN, Hilden, Germany). Then, libraries were sequenced on an IonProton system, and variants were called by Torrent Suite Software. Variants were outputted in VCF format.

\section{Annotation}

Variants were annotated with dbSNP rs identifiers (rs ID, NCBI dbSNP build 147), allele frequencies, and Combined Annotation Dependent Depletion (CADD) scores (Kircher et al., 2014) by using the ANNOVAR program (http:// annovar.openbioinformatics.org/). (Wang et al., 2010) For CADD scores, variants were annotated with CADD version 1.3 (cadd13). For allele frequencies in public databases, we used data from the 1000 Genomes Project (1000g2015aug), Exome Sequencing Project (esp6500siv2_all), and Exome Aggregation Consortium (exac03). We also annotated allele frequencies in the Japanese population by using the $2 \mathrm{KJPN}$ database (https://ijgvd.megabank. tohoku.ac.jp/) from TMM.

\section{Gene expression data}

We used gene expression data from the Genotype-Tissue Expression (GTEx) project (https://gtexportal.org/). Median reads per kilobase of exon per million mapped reads (RPKM) data of 13 brain regions from GTEx Analysis V6 were averaged for each gene and used for the variantfiltering step.

\section{Primers and probes}

All primers for PCR reactions, Sanger sequencing, and invader assays were synthesized commercially (Fasmac, Kanagawa, Japan). Primers were designed using the Primer3Plus program (http://primer3plus.com/cgi-bin/dev/ primer3plus.cgi).

\section{Sanger sequencing}

PCR was performed using AmpliTaq Gold 360 DNA Polymerase (Thermo Fisher Scientific). Purified PCR fragments were sequenced using the BigDye Terminator v3.1 Cycle Sequencing Kit and an ABI 3100 Genetic Analyzer (Thermo Fisher Scientific).

\section{Genotyping}

We genotyped candidate variants using a multiplex PCR invader assay (Third Wave Technologies, Madison, WI) (Ohnishi et al., 2001) by means of a QuantStudio 7 Flex Real-Time PCR System (Thermo Fisher Scientific) for NCGG and Niigata samples or ABI7900HT Fast Real-Time PCR System (Applied Biosystems) for RIKEN samples. For part of the first cohort control for the two loci (rs572750141 and rs531355933), we also obtained the genotyped data from 1765 in-house whole-genome sequences at RIKEN and the whole-genome sequence data of 3554 Japanese individuals at TMM (3.5KJPN, Integrative Japanese Genome Variation; https://ijgvd.megabank. tohoku.ac.jp/) (Additional file 1: Table S3).

\section{Construction of plasmid vectors}

Each PCR product for wild-type and G186R variant SHARPIN was prepared by using complementary DNA synthesized based on mRNA extracted from the buffy coat of the patients analyzed in this study and cloned into pCMV-Myc vector. All inserted sequences were confirmed by Sanger sequencing.

\section{Luciferase assay}

HEK293 cells were transfected with the luciferase reporter plasmid pGL4.32[luc2P/NF-kB-RE/Hygro] (Promega, Madison, WI), and stably expressing cells were selected by hygromycin. Twenty-four hours before transfection, cells were plated on 96 -well plates $\left(1.5 \times 10^{4}\right.$ cells/well). Transfection with plasmids was performed using FuGENE ${ }^{\oplus} \mathrm{HD}$ Transfection Reagent (Promega). Twenty-four hours after transfection, cells were treated with tumor necrosis factor- $\alpha$ (TNF- $\alpha, 20 \mathrm{ng} / \mathrm{ml}$ ) (Wako, Osaka, Japan) for $5 \mathrm{~h}$. Then, luciferase activity was measured by using a Nano-Glo $^{\circ} \quad$ Dual-Luciferase $^{\curvearrowleft}$ Reporter Assay System (Promega). Each experiment was independently performed three times with five replicates of each sample.

\section{Immunocytochemistry}

HEK293 cells were seeded at a density of $2.0 \times 10^{4}$ cells/ well on BioCoat ${ }^{\mathrm{TM}}$ Poly-D-Lysine 4-well Culture Slides (Corning, NY, USA), cultured for $24 \mathrm{~h}$, and transfected with wild-type and G186R Myc-SHARPIN plasmids. Twenty-four hours after transfection, cells were fixed and incubated with Anti-Myc-tag mAb-Alexa Fluor ${ }^{\circledR} 488$ (MBL, Nagoya, Japan) according to the manufacturer's protocol. Then, the slides were mounted with SlowFade ${ }^{\text {тm }}$ Diamond Antifade Mountant with DAPI (Thermo Fisher Scientific) and fluorescence images were obtained on a BIOREVO BZ-9000 fluorescence microscope (Keyence, Osaka, Japan).

\section{Statistical analysis}

All statistical analyses were performed using $\mathrm{R}$ software (version 3.2.4). For calculation of the odds ratio and 95\% confidence interval, vcd package (version 1.4.3) was used in R. Meta-analyses of two cohort sets were performed by using the Mantel-Haenszel $X^{2}$ test. For luciferase assay experiments, a Student's t-test was conducted to estimate the statistical difference in luciferase activity 
among cells transfected with mock (expressing myc), wild-type, and G186R plasmids.

\section{Results}

Step-by-step filtering to identify rare risk variants

We performed whole-exome sequencing analyses of 202 LOAD patients lacking the APOE $\varepsilon 4$ risk allele (Additional file 1: Table S1). In particular, we chose the samples so as to avoid mixed-type dementia (e.g., AD and vascular dementia and $\mathrm{AD}$ and dementia with Lewy bodies) as much as possible. All variants derived from exome sequencing analysis were annotated as denoted in the Methods section. First, we checked for known mutations in causal genes-APP, PSEN1, and PSEN2-for autosomal-dominant early-onset $\mathrm{AD}$ development. We did not detect any known early-onset $\mathrm{AD}$ pathogenic mutations in these genes. Then, all variants of the 202 patients were merged as one VCF-formatted list, which was followed by step-by-step filtering (Fig. 1). Annotated variants were divided into SNVs, indels, and others. In this study, we focused on SNVs because of the accuracy of variant calling. We selected variants with an $\mathrm{MAF}<0.01$ and those not reported in the annotated allele frequency data of each public database. Then, we excluded the variants found in our own exome sequence data of $176 \mathrm{NC}$ individuals (Additional file 1: Table S1).

The selected rare variants were filtered according to the deleteriousness of the corresponding gene products by means of CADD scores. The distribution of the CADD scores of the variants is shown in Additional file 1: Figure S1. Then, variants with a highly deleterious, scaled $\mathrm{C}$ score $>20$ were selected. In these steps, we focused on 21,084 variants.

Next, because the brain is an important tissue for $\mathrm{AD}$ progression, we excluded genes with low expression in the brain by using expression data from the GTEx database. First, we chose 10,461 genes with 21,084 variants. Then, we selected 8490 genes with an RPKM $>0$ and excluded 301 genes with a z-score $<-1.96$ (Additional file 1: Figure S2). Ultimately, we chose 8189 genes with 16,837 variants and performed a further filtering step to narrow-down the candidate variants.

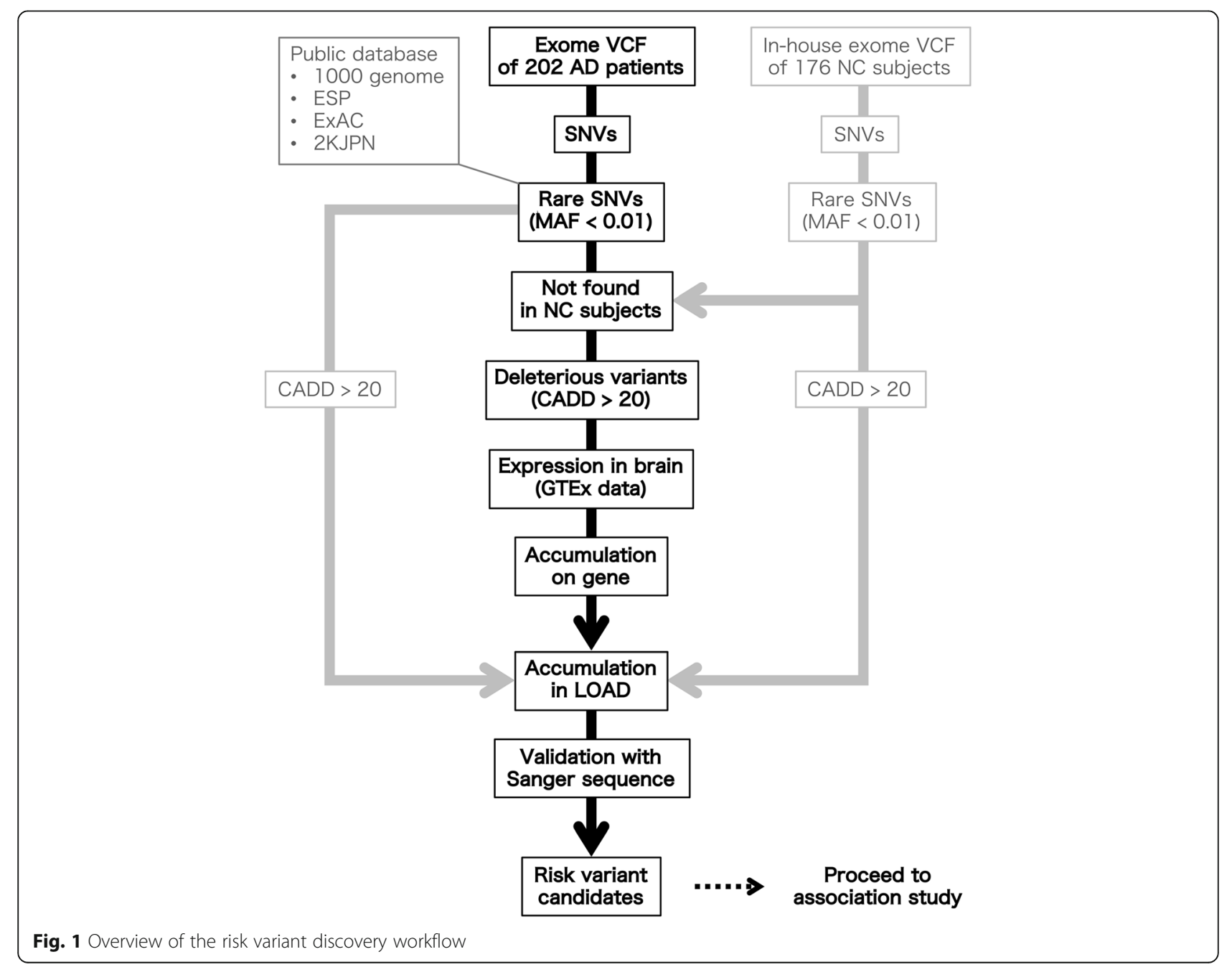


We hypothesized that the selected variants of each gene would accumulate against the expected substitution rate and the number of variants of each gene followed a Poisson distribution (Additional file 1: Figure S3). First, we selected 2557 genes with more than two AD patients who had the selected variants. Then, the number of variants of each gene was tested against the expected value from the Poisson distribution function, which was normalized according to CDS length. In this way, 10 genes with significant accumulation, a $q$-value $<0.01$ for FDR, were selected, as shown in Additional file 1: Table S5.

Furthermore, the accumulation of variants in LOAD patients against our in-house NC controls was evaluated by using a $X^{2}$ test. We found a significant accumulation of variants $(q<0.05)$ in four genes: SHARPIN, PXN, TYK2, and ZNF786 (Additional file 1: Table S6). Then, we confirmed 23 variants in these four genes by using Sanger sequencing; seven variants were non-polymorphic, whereas the others were consistent with exome data. Thus, we selected 16 variants in four genes as the risk factor candidates.

\section{Association study of candidate variants in the first cohort}

To evaluate the associations of the variants found in the exome sequencing analysis with LOAD, we genotyped 16 candidate variants with staged samples, including LOAD cases and NC controls, from the NCGG Biobank. In the first sample set with 1102 cases and 592 controls, nine variants were found in the $\mathrm{NC}$ controls, and we further genotyped the other seven variants in an additional 1079 case and 327 control samples. As shown in Table 1, all seven variants were not found in NC controls, whereas SHARPIN (rs572750141, p.Gly186Arg) and TYK2 (rs531355933, p.Val164Met) variants were found in LOAD patients. Then, we assessed the associations of these two variants with LOAD using joint analysis and by increasing the number of samples to 2383 for LOAD cases and to 13,973 for controls (Additional file 1: Table S3). The analysis with Bonferroni correction showed a significant association of the SHARPIN variant with LOAD (odds ratio $=8.4)($ Table 2$)$.

\section{Replication study and meta-analysis}

Next, we implemented a replication study with a second cohort set (2180 LOAD cases and $2486 \mathrm{NC}$ controls) recruited from Niigata University (Additional file 1: Table S4). Carriers of the SHARPIN variant were too rare in both case and control groups to evaluate the significance of differences in this cohort (Table 2). However, when we meta-analyzed these data, the SHARPIN variant showed a significant risk (corrected $P=8.05 \times 10^{-5}$ ) with a large effect size (odds ratio of 6.1), both higher than those of the TREM2 variant in an Icelandic cohort. (Jonsson et al., 2013) The TYK2 variant showed no significance.

\section{Functional analysis of the LOAD-associated variant}

To investigate how the LOAD-associated variant found in this study is involved in LOAD pathogenesis, we analyzed the effects of the SHARPIN G186R variant protein. First, because SHARPIN regulates inflammatory and immune responses through activation of the NF- $\mathrm{kB}$ pathway, we examined whether the variant protein affects NF- $\mathrm{kB}$ activity by means of a luciferase assay. As shown in Fig. 2a, NF-kB activity was significantly decreased when the variant-type SHARPIN-Myc-SHARPIN (G186R)-was expressed in HEK293 cells compared with wild-type Myc-SHARPIN.

We also hypothesized that the amino acid substitution might possibly alter the cellular localization of the protein. Thus, we used immunocytochemistry to examine the cellular localization of SHARPIN, ectopically expressed in HEK293 cells. As shown in Fig. 2b and c and Additional file 1: Figure S4, the G186R variant showed uneven lumps of SHARPIN granules in HEK293 cells, whereas the wild-type was uniformly distributed in the cytosol and had no such accumulations.

\section{Discussion}

We conducted whole-exome sequencing of 202 Japanese LOAD patients without APOE $\varepsilon 4$ alleles and found a novel coding rare variant (rs572750141, NM_0309 74.3:p.Gly186Arg) of SHARPIN that was significantly

Table 1 Seven candidate risk variants for LOAD

\begin{tabular}{|c|c|c|c|c|c|c|}
\hline \multirow[t]{2}{*}{ Position (hg19) } & \multirow[t]{2}{*}{ Gene } & \multirow[t]{2}{*}{ Ref./Alt. } & \multirow[t]{2}{*}{ Protein } & \multirow[t]{2}{*}{ dbSNP ID } & \multicolumn{2}{|c|}{ No. of carriers/No. determined } \\
\hline & & & & & LOAD & NC \\
\hline 12:120651694 & PXN & $\mathrm{T} / \mathrm{C}$ & p.Tyr487Cys & rs980452538 & $0 / 2176$ & 0/909 \\
\hline 8:145154709 & SHARPIN & $\mathrm{C} / \mathrm{T}$ & p.Gly186Arg & rs572750141 & $9 / 2172$ & 0/916 \\
\hline $8: 145154228$ & SHARPIN & $C / T$ & p.Gly292Arg & rs774802799 & $0 / 2183$ & 0/917 \\
\hline 19:10477232 & TYK2 & $C / T$ & p.Val164Met & rs531355933 & $3 / 2181$ & 0/916 \\
\hline 7:148769094 & ZNF786 & $C / T$ & p.Arg257His & rs771874615 & $0 / 2179$ & 0/914 \\
\hline 7:148767975 & ZNF786 & $\mathrm{T} / \mathrm{C}$ & p.Tyr630Cys & rs762922242 & $0 / 2180$ & 0/915 \\
\hline 7:148767897 & ZNF786 & $\mathrm{C} / \mathrm{T}$ & p.Gly656Asp & NA & $0 / 2175$ & 0/914 \\
\hline
\end{tabular}


Table 2 Associations of the two variants in SHARPIN and TYK2 with the risk of LOAD

\begin{tabular}{|c|c|c|c|c|c|c|c|c|c|c|c|c|c|}
\hline \multirow[t]{2}{*}{ dbSNP ID } & \multirow[t]{2}{*}{ Chr. } & \multirow[t]{2}{*}{ Gene } & \multirow[t]{2}{*}{ Phase } & \multicolumn{2}{|c|}{ Number of samples } & \multicolumn{2}{|c|}{ Number of variants } & \multicolumn{2}{|l|}{ MAF } & \multirow[t]{2}{*}{ OR } & \multirow[t]{2}{*}{$95 \% \mathrm{Cl}$} & \multirow[t]{2}{*}{$P$} & \multirow[t]{2}{*}{$P$ (Bonferroni) $^{b}$} \\
\hline & & & & Cases & Controls & Cases & Controls & Cases & Controls & & & & \\
\hline \multirow[t]{3}{*}{ rs572750141 } & 8 & SHARPIN & 1st cohort & 2383 & 13973 & 10 & 7 & 0.002 & 0.0003 & 8.4 & $3.2-22.1$ & $2.28 \times 10^{-7}$ & $1.6 \times 10^{-6}$ \\
\hline & & & 2nd cohort & 2180 & 2486 & 1 & 1 & 0.0002 & 0.0002 & 1.14 & $0.02-89.5$ & 1 & 1 \\
\hline & & & Combined $^{a}$ & 4563 & 16459 & 11 & 8 & 0.001 & 0.0002 & 6.1 & $2.4-15.5$ & $1.15 \times 10^{-5}$ & $8.05 \times 10^{-5}$ \\
\hline \multirow[t]{3}{*}{ rs531355933 } & 19 & TYK2 & 1st cohort & 2379 & 13973 & 4 & 5 & 0.0008 & 0.0002 & 4.7 & $1.3-17.5$ & 0.011 & 0.77 \\
\hline & & & 2nd cohort & 2180 & 2486 & 3 & 4 & 0.0007 & 0.0008 & 0.86 & $0.13-5.1$ & 1 & 1 \\
\hline & & & Combined $^{\mathrm{a}}$ & 4559 & 16459 & 7 & 9 & 0.0008 & 0.0003 & 1.93 & $0.71-5.2$ & 0.26 & 1 \\
\hline
\end{tabular}

ID; Identifier, Chr; Chromosome, MAF; Minor allele frequency, OR; Odds ratio, $\mathrm{Cl}$; Confidence interval

${ }^{\mathrm{a}} P$ value was calculated by Mantel-Haenszel test

${ }^{\mathrm{b}}$ Bonferroni-corrected $P$ value, calculated by setting the number of tests to seven

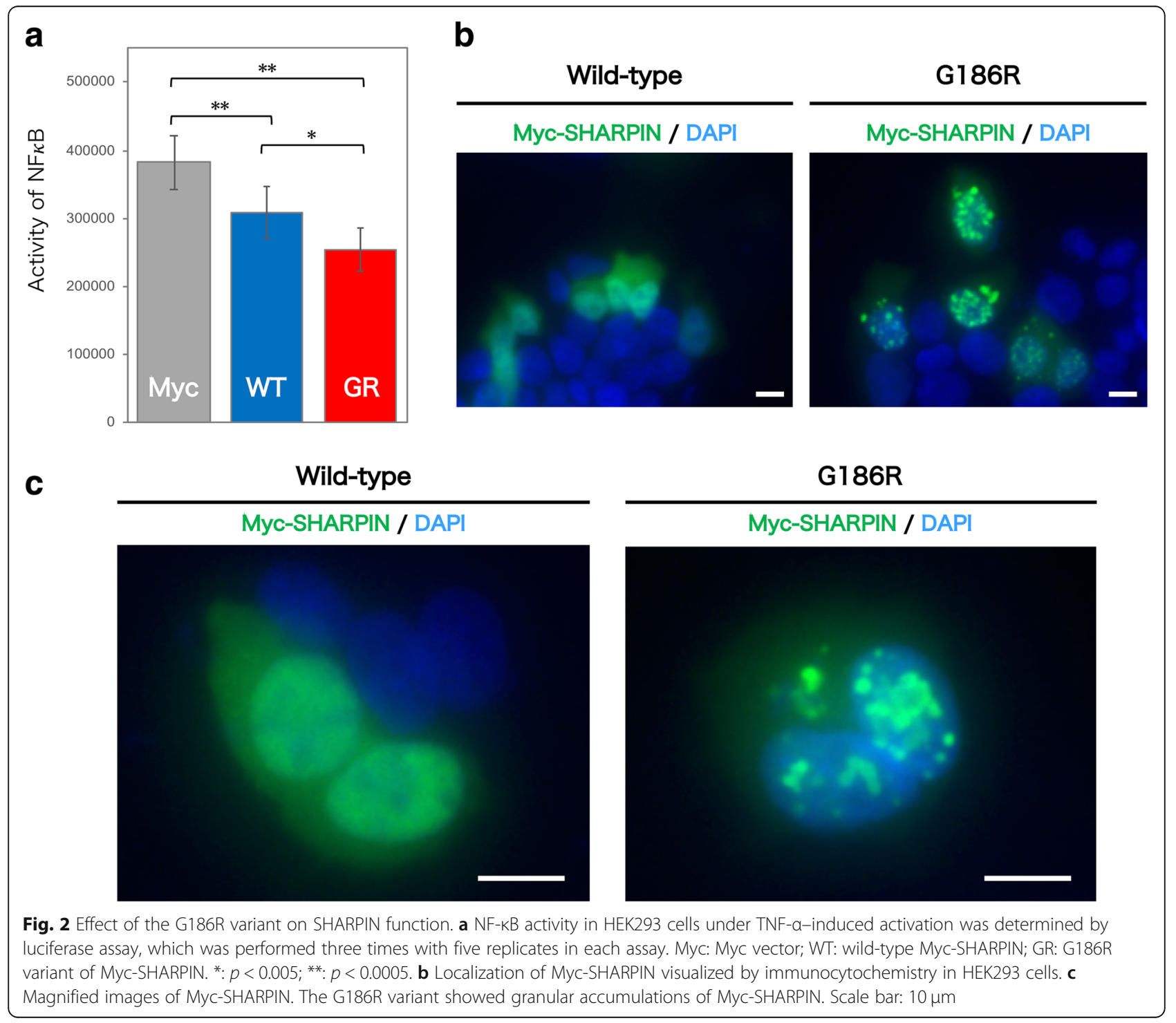


associated with a high odds ratio with increased risk of LOAD in the Japanese population (Table 2). However, the results of the association study obtained here have some limitations. Although the meta-analysis showed a significant difference based on sufficient power, only two rs572750141 carriers were found in the second cohort, one case and one control. Therefore, the statistical significance needs to be clarified in another Japanese cohort, and an additional large cohort is required to reduce statistical fluctuations as much as possible. Additionally, our strategy for filtering the exome variants might have some risk gene selection biases due to the step-by-step filtering approach applied. In particular, we focused on genes even slightly expressed in brain tissue. Therefore, genes specifically expressed in peripheral tissues that might nonetheless act as risk factors may have been missed.

SHARPIN is a postsynaptic density protein. (Lim et al., 2001) It is also a component of the linear ubiquitin chain assembly complex (LUBAC). LUBAC regulates inflammation through activation of the NF- $\mathrm{kB}$ pathway by conjugating linear ubiquitin chains to IKBKG/NEMO and RIPK1. (Gerlach et al., 2011; Ikeda et al., 2011; Tokunaga et al., 2011) Chronic proliferative dermatitis mutation (cpdm) mice, which are spontaneous Sharpin-deficient mutant mice, develop chronic proliferative dermatitis from 3 to 5 weeks of age. (Gallardo Torres et al., 1995; Gijbels et al., 1996; HogenEsch et al., 1993; HogenEsch et al., 1999; Seymour et al., 2006) The lifespan of this mutant mouse is shortened, and the mice show a decreased body size and a characteristic progressive dermatitis with alopecia. In this study, we found that the G186R variant affects the localization of SHARPIN proteins (Fig. $2 \mathrm{~b}$ and $\mathrm{c}$ and Additional file 1: Figure S4) and attenuates TNF- $\alpha$-induced activation of downstream NF-kB (Fig. 2a). The glycine residue at position 186 of SHARPIN is highly conserved among species (Additional file 1: Figure S5), indicating the importance of this amino acid in cellular function. In fact, this single amino acid substitution drastically altered the cellular localization of SHARPIN. Although it remains to be clarified whether the uneven lumps of SHARPIN G186R granules were due to their uptake into certain organelles or the formation of immunoreactive aggregates, the reduction in $\mathrm{NF}-\mathrm{kB}$ activity induced by the variant might be involved in this aberrant localization.

In recent studies of rare TREM2 variants associated with LOAD with large effect sizes, attenuation of the inflammatory response and immune function in the nervous system, particularly in microglia, was reported to contribute to $\mathrm{AD}$ onset. (Abbott, 2018; Sims et al., 2017; Wang et al., 2015; Wendeln et al., 2018) SHARPIN also functions as an inflammatory/immune mediator through activation of $\mathrm{NF}-\mathrm{kB}$, a central mediator of the inflammatory/immune cascade. Our in vitro experiments with the SHARPIN variant revealed its aberrant localization and additional attenuated NF- $\mathrm{kB}$ activity, indicating that the variant possibly plays a role in the development of LOAD through aberrant inflammatory/immune functions in the nervous system, which may be similar to the function of the TREM2 variant. Furthermore, a recent computational study indicated the association between the SHARPIN gene and AD. (Lancour et al., 2018) In this report, Lancour et al. developed a novel integrated GWAS risk score with a network diffusion approach. They tested this approach on a large GWAS dataset from Caucasians with AD and identified SHARPIN as a possible AD risk gene with the second ranked risk score. In addition, a report posted on a preprint server (Soheili-Nezhad et al., 2017) noted that a nonsynonymous variant of SHARPIN (rs34173062: p.Ser17Phe) correlated with amygdala atrophy as an endophenotype in $\mathrm{AD}$. Furthermore, RIPK1, which is located downstream of LUBAC, mediates a disease-associated microglial response in AD. (Ofengeim et al., 2017) Taken together, these results suggest that our novel SHARPIN variant associates with LOAD onset by affecting inflammation and/or immune function in the nervous system, although the detailed mechanisms underlying the LOAD pathogenesis remain to be clarified.

\section{Conclusions}

We identified a novel coding rare variant of SHARPIN as a genetic factor associated with risk of LOAD with a large effect size in the Japanese population via whole-exome sequencing of 202 patients. Our initial sample size is relatively modest for the field of LOAD genetic research in recent years but our pipeline has found a convincing risk factor, indicating the possible appropriateness of our strategy to identify rare disease variants with strong effects. Accordingly, the pipeline applied in this study might be effective for other common diseases. This is the first in vitro demonstration that the rare nonsynonymous variant of SHARPIN associated with LOAD onset has critical physiological effects. Further genetic investigations with large East Asian populations may reveal the association between SHARPIN and LOAD globally.

Finally, LOAD is a problem not only for patients, but also those that care for them and is also a leading cause of death in many countries. We believe that clarification of the genetic architecture and the implicated pathways helps to explain the pathogenesis of LOAD and will contribute to future medical and pharmaceutical research efforts to develop precision medicine for this common but serious disease.

\section{Additional file}

Additional file 1: Figure S1. Distribution of CADD scores. Figure S2. Gene expression in the brain. Figure S3. Distribution of the number of 
variants in genes. Figure S4. Detailed images of the immunocytochemistry shown in Fig. 2b. Figure S5. G186 of SHARPIN is highly conserved among species. Protein sequences of SHARPIN for each species were obtained from UniProt and the region near G186 was compared. Table S1. Samples used for exome sequencing. Table S2. Demographic features of the NCGG samples genotyped. Table S3. Samples used in the first association study of candidate AD risk variants. Table S4. Demographic features of the second cohort set used in the association study. Table S5. Ten genes with significantly accumulated variants. Table S6. Accumulation of variants in LOAD patients compared with NC controls. (PDF $3193 \mathrm{~kb}$ )

\section{Abbreviations}

CADD: Combined annotation dependent depletion; gnomAD: Genome Aggregation Database; GTEx: Genotype-Tissue Expression; LOAD: Late-onset Alzheimer's disease; MAF: Minor allele frequency; NC: Normal cognitive function; NF-kB: Nuclear factor kappa B subunit 1; NIA-AA: National Institute on Aging and the Alzheimer's Association; RPKM: Reads per kilobase of exon per million mapped reads; SHARPIN: SHANK-associated RH domain interactor; TNF-a: Tumor necrosis factor-a; TREM2: Triggering receptor expressed on myeloid cells 2

\section{Acknowledgements}

We thank the staff members of the NCGG Medical Genome Center, the Laboratory for Cardiovascular Diseases at RIKEN, and the Department of Molecular Genetics at Niigata University for their contribution to this study.

\section{Funding}

This work was partly supported by The Research Funding for Longevity Sciences (29-45) from the National Center for Geriatrics and Gerontology (to K.O.); grants from the Japan Agency for Medical Research and Development (AMED) (Grant Number JP18kk0205009 to S.N., Grant Number JP18kk0205012 to S.N., and Grant Number JP19dk0207028 to T.I.); and a grant for Research on Dementia from the Japanese Ministry of Health, Labor and Welfare (to K.O.).

\section{Availability of data and materials}

The datasets used and/or analyzed during the current study are available from the corresponding author on reasonable request.

\section{Authors' contributions}

YA performed all of the core experiments and analyses and wrote the manuscript. RM and TM performed genotyping and analysis. SN managed all samples from the NCGG Biobank. DS performed statistical analysis to narrowdown the risk variants. KI managed the DNA samples and genotyping data at RIKEN. AM, NH and TI managed the DNA samples for the second cohort at Niigata University and performed genotyping. KO supervised this study. All authors read and approved the final manuscript.

\section{Ethics approval and consent to participate}

All subjects provided written informed consent. Ethics approval was obtained from the ethics review board at NCGG and of each institute.

\section{Consent for publication}

Not applicable.

\section{Competing interests}

The authors declare that they have no competing interests.

\section{Publisher's Note}

Springer Nature remains neutral with regard to jurisdictional claims in published maps and institutional affiliations.

\section{Author details}

'Division for Genomic Medicine, Medical Genome Center, National Center for Geriatrics and Gerontology, Obu, Aichi, Japan. ${ }^{2}$ Department of Medical Science Mathematics, Medical Research Institute, Tokyo Medical and Dental University, Tokyo, Japan. ${ }^{3}$ Laboratory for Medical Science Mathematics, RIKEN Center for Integrative Medical Sciences, Yokohama, Japan. ${ }^{4}$ Department of Molecular Genetics, Brain Research Institute, Niigata University, Niigata, Japan. ${ }^{5}$ Laboratory for Cardiovascular Diseases, RIKEN Center for Integrative Medical Sciences, Yokohama, Japan. ${ }^{6}$ Medical Genome Center, National Center for Geriatrics and Gerontology, Obu, Aichi, Japan.
Received: 23 January 2019 Accepted: 6 May 2019

Published online: 20 June 2019

\section{References}

Abbott A. Is 'friendly fire' in the brain provoking Alzheimer's disease? Nature. 2018;556:426-8.

Albert MS, DeKosky ST, Dickson D, Dubois B, Feldman HH, Fox NC, et al. The diagnosis of mild cognitive impairment due to Alzheimer's disease: recommendations from the National Institute on Aging-Alzheimer's association workgroups on diagnostic guidelines for Alzheimer's disease. Alzheimers Dement. 2011;7:270-9.

Bagyinszky E, Youn YC, An S, Kim S. The genetics of Alzheimer's disease. Clin Interv Aging. 2014;9:535-51.

Gallardo Torres HI, Gijbels MJJ, HogenEsch H, Kraal G. Chronic proliferative dermatitis in mice: neutrophil-endothelium interactions and the role of adhesion molecules. Pathobiology. 1995;63:341-7.

Gatz M, Reynolds CA, Fratiglioni L, Johansson B, Mortimer JA, Berg S, et al. Role of genes and environments for explaining Alzheimer disease. Arch Gen Psychiatry. 2006;63:168-74.

Gerlach B, Cordier SM, Schmukle AC, Emmerich CH, Rieser E, Haas TL, et al. Linear ubiquitination prevents inflammation and regulates immune signalling. Nature. 2011:471:591-6.

Gijbels MJ, Zurcher C, Kraal G, Elliott GR, HogenEsch H, Schijff G, et al. Pathogenesis of skin lesions in mice with chronic proliferative dermatitis (cpdm/cpdm). Am J Pathol. 1996;148:941-50.

Guerreiro R, Wojtas A, Bras J, Carrasquillo M, Rogaeva E, Majounie E, et al. TREM2 variants in Alzheimer's disease. N Engl J Med. 2013;368:117-27.

HogenEsch H, Gijbels MJ, Offerman E, van Hooft J, van Bekkum DW, Zurcher C. A spontaneous mutation characterized by chronic proliferative dermatitis in C57BL mice. Am J Pathol. 1993;143:972-82.

HogenEsch H, Janke S, Boggess D, Sundberg JP. Absence of Peyer's patches and abnormal lymphoid architecture in chronic proliferative dermatitis (cpdm/ cpdm) mice. J Immunol. 1999;162:3890-6.

Ikeda F, Deribe YL, Skånland SS, Stieglitz B, Grabbe C, Franz-Wachtel M, et al. SHARPIN forms a linear ubiquitin ligase complex regulating NF-KB activity and apoptosis. Nature. 2011;471:637-41.

Jonsson T, Stefansson H, Steinberg S, Jonsdottir I, Jonsson PV, Snaedal J, et al. Variant of TREM2 associated with the risk of Alzheimer's disease. N Engl J Med. 2013:368:107-16.

Kircher M, Witten DM, Jain P, O'Roak BJ, Cooper GM, Shendure J. A general framework for estimating the relative pathogenicity of human genetic variants. Nat Genet. 2014;46:310-5.

Lambert J-C, Ibrahim-Verbaas CA, Harold D, Naj AC, Sims R, Bellenguez C, et al. Meta-analysis of 74,046 individuals identifies 11 new susceptibility loci for Alzheimer's disease. Nat Genet. 2013;45:1452-8.

Lancour D, Naj A, Mayeux R, Haines JL, Pericak-Vance MA, Schellenberg GD, et al. One for all and all for one: improving replication of genetic studies through network diffusion. PLoS Genet. 2018;14:e1007306.

Lim S, Sala C, Yoon J, Park S, Kuroda S, Sheng M, et al. Sharpin, a novel postsynaptic density protein that directly interacts with the shank family of proteins. Mol Cell Neurosci. 2001;17:385-97.

McKhann GM, Knopman DS, Chertkow H, Hyman BT, Jack CR, Kawas CH, et al. The diagnosis of dementia due to Alzheimer's disease: recommendations from the National Institute on Aging-Alzheimer's association workgroups on diagnostic guidelines for Alzheimer's disease. Alzheimers Dement. 2011;7:263-9.

Miyashita A, Wen Y, Kitamura N, Matsubara E, Kawarabayashi T, Shoji M, et al. Lack of genetic association between TREM2 and late-onset Alzheimer's disease in a Japanese population. J Alzheimers Dis. 2014;41:1031-8.

Ofengeim D, Mazzitelli S, Ito Y, DeWitt JP, Mifflin L, Zou C, et al. RIPK1 mediates a disease-associated microglial response in Alzheimer's disease. Proc Natl Acad Sci. 2017;114:E8788-97.

Ohnishi Y, Tanaka T, Ozaki K, Yamada R, Suzuki H, Nakamura Y. A highthroughput SNP typing system for genome-wide association studies. J Hum Genet. 2001;46:471-7.

Saji N, Niida S, Murotani K, Hisada T, Tsuduki T, Sugimoto T, et al. Analysis of the relationship between the gut microbiome and dementia: a cross-sectional study conducted in Japan. Sci Rep. 2019;9:1008.

Scheltens P, Blennow K, Breteler MMB, de Strooper B, Frisoni GB, Salloway S, et al. Alzheimer's disease. Lancet. 2016;388:505-17. 
Seymour R, Sundberg JP, Esch HH. Abnormal lymphoid organ development in immunodeficient mutant mice. Vet Pathol. 2006;43:401-23.

Sims R, van der Lee SJ, Naj AC, Bellenguez C, Badarinarayan N, Jakobsdottir $J$, et al. Rare coding variants in PLCG2, ABI3, and TREM2 implicate microglial-mediated innate immunity in Alzheimer's disease. Nat Genet. 2017:49:1373-84.

Soheili-Nezhad S, Jahanshad N, Tajer A, Guelfi S, Khosrowabadi R, Pouretemad H, et al. Amygdalar atrophy as the genetically mediated hub of limbic degeneration in Alzheimer's disease. bioRxiv. 2017:196410.

Tokunaga F, Nakagawa T, Nakahara M, Saeki Y, Taniguchi M, Sakata S, et al. SHARPIN is a component of the NF-KB-activating linear ubiquitin chain assembly complex. Nature. 2011;471:633-6.

Van Cauwenberghe C, Van Broeckhoven C, Sleegers K. The genetic landscape of Alzheimer disease: clinical implications and perspectives. Genet Med. 2016:18:421-30.

Wang K, Li M, Hakonarson H. ANNOVAR: functional annotation of genetic variants from high-throughput sequencing data. Nucleic Acids Res. 2010;38:e164.

Wang Y, Cella M, Mallinson K, Ulrich JD, Young KL, Robinette ML, et al. TREM2 lipid sensing sustains the microglial response in an Alzheimer's disease model. Cell. 2015;160:1061-71.

Wendeln A-C, Degenhardt K, Kaurani L, Gertig M, Ulas T, Jain G, et al. Innate immune memory in the brain shapes neurological disease hallmarks. Nature. 2018;556:332-8

Ready to submit your research? Choose BMC and benefit from:

- fast, convenient online submission

- thorough peer review by experienced researchers in your field

- rapid publication on acceptance

- support for research data, including large and complex data types

- gold Open Access which fosters wider collaboration and increased citations

- maximum visibility for your research: over $100 \mathrm{M}$ website views per year

At BMC, research is always in progress.

Learn more biomedcentral.com/submissions 